\title{
Determinação da massa equivalente de hidrogênio amina em endurecedores do tipo amina alifática por titulação potenciométrica
}

\author{
GONZÁLEZ GARCIA, F.; MATUSALÉM, F.; LEYVA, M.E.; QUEIROZ, A.A.A. \\ Laboratório de Biomateriais-DFQ/ICE/UNIFEI Av. BPS,1303, Pinheirinho CEP: 37500-903, Itajubá, MG. \\ e-mail: fili@unifei.edu.br, fmatusalem@yahoo.com.br, mariae@unifei.edu.br, alencar@unifei.edu.br
}

\section{RESUMO}

Um conjunto de endurecedores de resinas epoxídicas do tipo aminas alifáticas foram caracterizados por titulação potenciométrica. Os endurecedores correspondem a compostos de cadeia linear: etilenoamina (EDA), dietilenotriamina (DETA), trietilenotetramina (TETA), tetraetilenopentamina (TEPA), e compostos de cadeia cíclica: 1-(2-aminoetil)piperazina (AEP), isoforondiamina (IPD) e 4,4-diamino-3,3'dimetilciclohexilmetano (3DCM). Determinou-se a massa equivalente de hidrogênio amina (MEHA) de cada composto de modo indireto por reação de protonação dos nitrogênios do tipo amina utilizando-se solução de ácido clorídrico padrão $(\mathrm{HCl})$, baseado na técnica de titulação potenciométrica em meio aquoso. $\mathrm{O}$ procedimento fornece também o valor amina (miligramas de $\mathrm{KOH}$ por grama de endurecedor). Foi ainda determinado o pH da solução aquosa $(1 \mathrm{~g}$ em $100 \mathrm{~mL}$ de água destilada ), afim de avaliar o grau de basicidade. Os gráficos obtidos de $\mathrm{pH}$ vs Vol. de solução de $\mathrm{HCl}$, revelaram a existência de um ou dois saltos para os compostos. Este comportamento foi relacionado às reações de protonação dos nitrogênios primários, secundários e terciários presentes nos compostos. Os valores experimentais da MEHA obtidos foram muito próximos dos valores teóricos calculados. A técnica utilizada é simples e pode ser utilizada em qualquer laboratório de controle.

Palavras-chaves: aminas alifáticas, resina epoxídica, titulação potenciométrica.

\section{Determination of the amine hydrogen equivalent weight in aliphatic amine epoxide hardeners by a method of potentiometric titration}

\section{ABSTRACT}

A set of aliphatic amine epoxide hardeners were characterized. The method used is based on potentiometric titration. The hardeners correspond to compounds of linear structure: ethyleneamine (EDA), diethylenetriamine (DETA), triethylenetetramine (TETA), tetraethylenepentamine (TEPA), and compounds of cyclic structure: 1 - (2-aminoethyl) piperazine (AEP), isoforondiamine (IPD) and 4,4'-diamine-3, 3'dimethylciclohexilmetano (3DCM). The amine hydrogen equivalent weight (MEHA) was determined for each compound by an indirect method. The method is a technique of potentiometric titration in water, the reaction of amine-type nitrogen protonation occurred using a standard solution of hydrochloric acid $(\mathrm{HCl})$. The procedure also provides value amine (milligrams of $\mathrm{KOH}$ per gram of hardener) for each compound. The $\mathrm{pH}$ of the aqueous solution ( $1 \mathrm{~g}$ in $100 \mathrm{ml}$ of water) was also determined in order to assess the degree of basicity. The graphics obtained from $\mathrm{pH}$ vs $\mathrm{Vol}$ solution of $\mathrm{HCl}$ revealed that there are one or two jumps for the compound. This behavior was related to the protonation reactions for primary, secondary and tertiary nitrogens present in the compound. The experimental values of MEHA were very close to the theoretical values calculated. The technique is simple and can be used in any laboratory of control.

Keywords: aliphatic amine, epoxy resin, potentiometric titration.

\section{INTRODUÇÃO}

As resinas epoxídicas são materiais termorrígidos amplamente utilizados em adesivos, matrizes para compósitos em aplicações na indústria elétrica e recobrimentos, entre outras aplicações. Isto é, resultado de suas excelentes propriedades adesivas, mecânicas, térmicas e elétricas, assim como da relativa facilidade no processamento dos artefatos. Quando estes materiais são destinados para aplicações especiais que solicitam alta durabilidade, é comum a revisão dos parâmetros da formulação para atingir propriedades otimizadas [1- 
3]. Os principais parâmetros incluem a utilização de uma proporção adequada de resina e endurecedor ou agente de cura, um programa de cura adequado que assegure a conversão máxima da reação de polimerização e o emprego de um tempo de manipulação que garanta uma correta utilização da formulação.

Entre as várias famílias de endurecedores utilizados para as resinas epoxídicas, as mais conhecidas e estudadas são as do tipo amina. Esta família é constituída por numerosos compostos polifuncionais com diferentes estruturas químicas, as quais têm em comum a presença de grupos amina do tipo, primária, secundária e terciária. Particularmente os sistemas que utilizam agentes de cura do tipo amina alifática funcionam à temperatura ambiente, sendo amplamente utilizados para formulações adesivas de uso geral. Estas formulações permitem um tempo de manipulação adequado o que garante a correta aplicação pratica. [4] Estes sistemas são geralmente bi-componentes, sendo que os produtos comerciais se apresentam em duas embalagens, um deles contendo a resina e o outro o endurecedor como os componentes ativos mais importantes da formulação. A fim de obter bom desempenho, proporções estequiométricas de ambos componentes devem ser utilizadas. Isto requer em primeiro lugar a determinação do equivalente de hidrogênio ativo do agente de cura e o equivalente epoxídico da resina.

Para a determinação do equivalente de hidrogênio ativo em aminas alifáticas simples, quer dizer, em aminas contendo grupo amina primária, esta determinação pode ser realizada de modo simples, usando técnica de titulação potenciométrica em meio aquoso, usando uma solução de ácido clorídrico padrão. Entretanto, quando na estrutura química e/ou na composição do agente de cura existe uma combinação de grupos amina primária, secundária e terciária a situação não resulta tão simples como no caso anterior, e na literatura não existe reporte para este caso particular. Existe uma metodologia internacional adotada para a determinação por separado do conteúdo de nitrogênio de grupo amina primária, secundaria e terciária para endurecedores do tipo amina [5]. Este método não fornece o equivalente de hidrogênio ativo para este tipo de compostos. Esta situação foi tratada em trabalho publicado anteriormente onde foi desenvolvido um procedimento para resolver este problema usando técnica de titulação potenciométrica em meio aquoso [6] .

Neste trabalho foi escolhida a técnica de titulação potenciométrica em meio aquoso tratada em trabalho anterior como foi mencionado para determinar a massa equivalente de hidrogênio ativo do tipo amina em uma família maior de agentes de cura do tipo aminas alifáticas. O procedimento fornece também o valor amina (miligramas de $\mathrm{KOH}$ por grama de endurecedor) e a concentração de nitrogênios de tipo amina presente na amostra $\left(\mathrm{g} \mathrm{eq}^{-1}\right)$ de acordo com o tipo de nitrogênio (primário, secundário e terciário). Utilizando então este procedimento, um conjunto de endurecedores de resinas epoxídicas foi caracterizado.

\section{MATERIAIS E MÉTODOS}

Os endurecedores selecionados correspondem a compostos de cadeia linear: etilenoamina (EDA), dietilenotriamina (DETA), trietilenotetramina (TETA), tetraetilenopentamina (TEPA) e compostos de cadeia cíclica: 1-(2-aminoetil)piperazina (AEP), isoforondiamina (IPD) e 4,4'-diamino-3,3'-dimetilciclohexilmetano (3DCM). Sendo comercializados pelas empresas, Dow Química do Brasil S.A. (TETA, grau técnico), SigmaAldrich do Brasil (EDA, DETA, AEP, IPD, 3DCM $\geq 99 \%$ P.A.), Acros do Brasil S.A.(TEPA, grau técnico). As características de cada composto são apresentadas na Tabela 1.

\section{RESULTADOS}

Os endurecedores EDA, DETA, TETA, e TEPA correspondem a etilenoaminas polifuncionais, com estruturas químicas alifáticas de cadeia linear. No caso, dois últimos compostos além da estrutura linear apresentam em sua composição compostos com estruturas químicas ramificadas e cíclicas (do tipo piperazina). [1, 7] Estes dois compostos de grau técnico apresentam diferentes massas moleculares e composições químicas diferentes. Uma característica importante que distingue bem o comportamento destes dois endurecedores é o fato de conterem nitrogênios amina do tipo primário, secundário e terciário [7]. Os agentes de cura AEP, IPD e 3DCM apresentam estruturas químicas alifáticas de cadeia cíclica. As Figuras 1 e 2 mostram as curvas potenciométricas correspondentes às titulações em meio aquoso dos agentes de cura estudados.

O método consiste em realizar titulações potenciométricas em meio aquoso, utilizando-se uma solução padronizada de ácido clorídrico, conforme metodologia anteriormente divulgada []]. Para este objetivo foi utilizado um pHmetro digital de fabricação alemã (WTW, modelo 330i). 
Tabela 1: Estrutura química e funcionalidade dos compostos analisados.

\begin{tabular}{|c|c|c|c|}
\hline Nome & Designação & Estrutura química & Functionalidade \\
\hline Etilenoamina & EDA & & 4,0 \\
\hline Dietilenotriamina & DETA & & 5,0 \\
\hline Trietilenotetramina & TETA & & 6,0 \\
\hline Tetraetilenopentamina & TEPA & & $\mathrm{NH}_{2}$ \\
\hline 1-(2-Aminoetil)piperazina & AEP & & 3,0 \\
\hline Isophorondiamina & IPD & & 4,0 \\
\hline $\begin{array}{l}\text { 4,4'-Diamino-3,3'-dimetil- } \\
\text { diciclohexilmethano }\end{array}$ & 3DCM & & 4,0 \\
\hline
\end{tabular}

A Tabela 2 mostra os valores da massa equivalente de hidrogênio ativo do tipo amina teórica, calculados a partir da estrutura molecular de cada composto, e os valores experimentais obtidos a partir das titulações. Nota-se uma boa concordância entre os valores teóricos e experimentais confirmando a eficácia do método usado. Dos agentes de cura estudados três deles apresentaram dois saltos na curva potenciométrica, DETA, TETA e TEPA. O primeiro salto foi atribuído às reações de protonação do ácido clorídrico com os nitrogênios primários, e o segundo salto às reações de protonação do ácido com os nitrogênios secundários e/ou terciários como foi mencionado anteriormente.

No caso, dos outros compostos foi evidente a existência de apenas um salto, devido ao fato de existir apenas nitrogênios do tipo primário, exceto no caso do endurecedor AEP onde existem também nitrogênios do tipo secundário e terciário e a curva potenciométrica deste composto mostrou a existência de apenas um salto. Este comportamento foi relacionado à possível sobreposição das reações de protonação dos nitrogênios secundários e terciários com o nitrogênio primário.

Tabela 2: Massa equivalente de hidrogênio do tipo amina $\left(\mathrm{g} \mathrm{eq}^{-1}\right)$ dos compostos.

\begin{tabular}{|c|c|c|c|c|}
\hline Endurecedor & $\begin{array}{c}\mathrm{g} \mathrm{eq}^{-1} \text { com } \mathrm{HCl} \\
\text { (teórico) }\end{array}$ & $\begin{array}{c}\mathrm{g} \mathrm{eq}^{-1} \text { com } \mathrm{HCl} \\
\text { (exp.)* }\end{array}$ & $\begin{array}{c}\text { Massa equivalente de } \\
\text { hidrogênio amina } \\
\text { (teórico) }\end{array}$ & $\begin{array}{c}\text { Massa equivalente de } \\
\text { hidrogênio amina } \\
\text { (exp.) }\end{array}$ \\
\hline EDA & 30,05 & $32,62 \pm 0,78$ & 15,02 & $16,31 \pm 1,24$ \\
\hline DETA & 34,39 & $34,19 \pm 0,05$ & 20,51 & $20,52 \pm 0,03$ \\
\hline TETA & 36,56 & $30,38 \pm 1,64$ & 24,37 & $20,25 \pm 1,09$ \\
\hline TEPA & 37,86 & $42,26 \pm 1,78$ & 27,04 & $30,18 \pm 1,15$ \\
\hline AEP & 64,60 & $65,71 \pm 0,47$ & 43,06 & $43,81 \pm 0,32$ \\
\hline IPD & 84,64 & $87,02 \pm 0,13$ & 42,32 & $43,51 \pm 0,06$ \\
\hline 3DCM & 119,21 & $127,80 \pm 1,43$ & 59,60 & $63,90 \pm 0,72$ \\
\hline
\end{tabular}

*(exp.), valores experimentais resultantes da média dos valores encontrados nos procedimentos de titulação realizados para cada composto em pelo menos seis análises. 

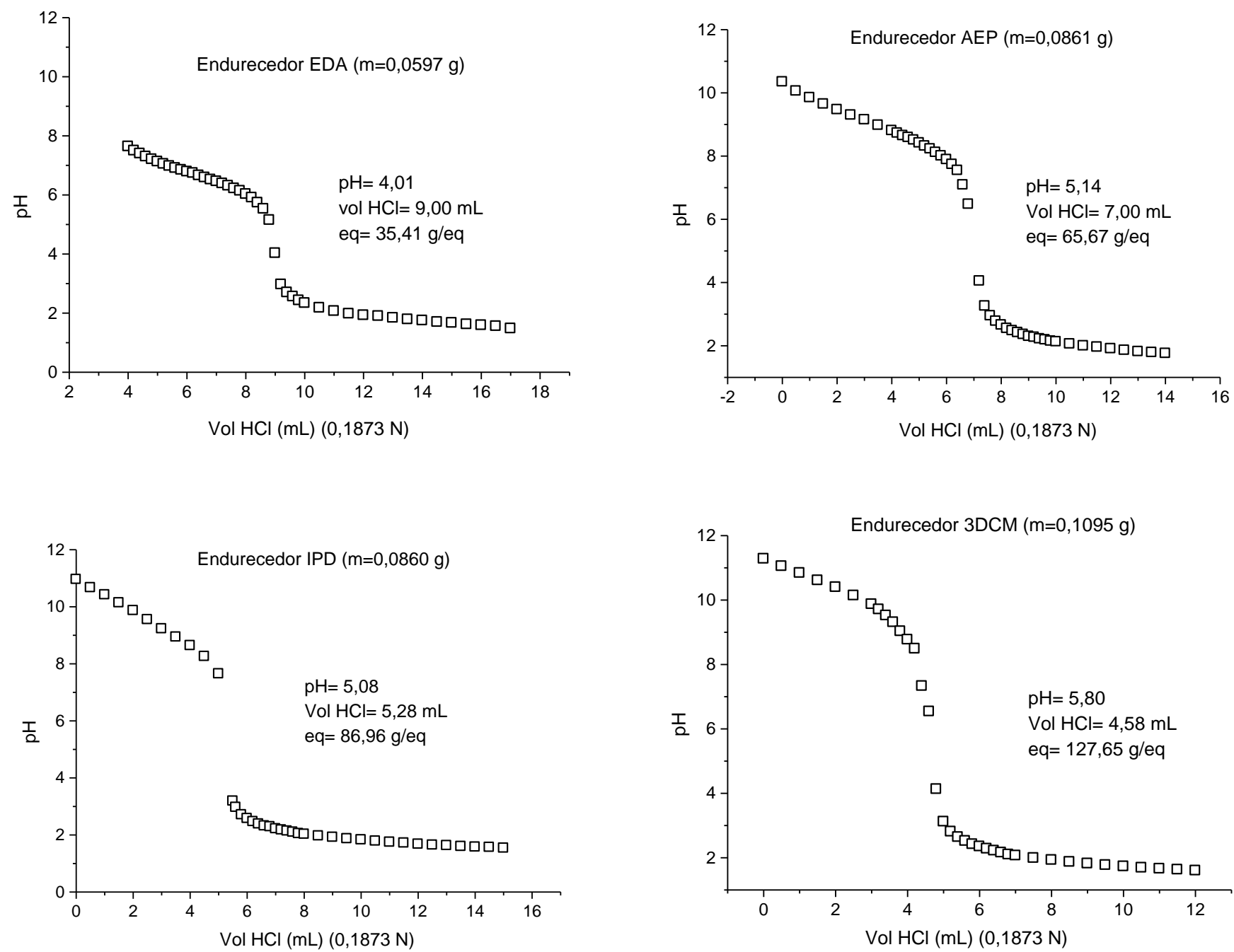

Figura 1: Curvas potenciométricas correspondentes a um processo de titulação em meio aquoso dos endurecedores EDA, AEP, IPD e 3DCM.

Na Tabela 3 é apresentado o valor amínico (miligramas de $\mathrm{KOH}$ por grama de composto) e o valor de pH da solução aquosa de 1 grama de agente de cura em $100 \mathrm{~mL}$ de água destilada. No caso, dos compostos DETA e TETA nota-se que o valor de $\mathrm{pH}$ nos dois pontos de equivalência, correspondentes ao $1^{\circ}$ salto e $2^{\circ}$ salto são muito semelhantes, embora o composto TETA seja um pouco mais básico como pode ser verificado a partir da comparação do valor amínico, embora os valores do pH da solução aquosa resultam muito semelhantes. Para o composto TETA foi verificado que os valores de $\mathrm{pH}$ onde ocorrem os dois saltos correspondentes aos pontos de equivalência são mais acentuados quando comparados ao composto TEPA. Isto foi relacionado à diferente composição química entre estes dois endurecedores onde o conteúdo de nitrogênio do tipo secundário e terciário resultam maiores no composto TEPA quando comparado ao TETA.[6] 
Tabela 3: Características físico-químicas dos agentes de cura

\begin{tabular}{|c|c|c|c|c|}
\hline \multirow[t]{2}{*}{ Endurecedor } & \multirow{2}{*}{$\begin{array}{c}\text { mg de } \\
\text { KOH/grama }\end{array}$} & \multicolumn{2}{|c|}{ pH pt. Eq. } & \multirow{2}{*}{$\begin{array}{l}\mathrm{pH} 1 \mathrm{~g} / 100 \mathrm{~mL} \\
\text { água destilada }\end{array}$} \\
\hline & & 1'salto & $2^{\circ}$ salto & \\
\hline EDA & $1726,7 \pm 127,0$ & $4,01 \pm 0,02$ & - & 11,55 \\
\hline DETA & $1641,2 \pm 24,6$ & $6,70 \pm 0,03$ & $2,85 \pm 0,06$ & 11,42 \\
\hline TETA & $1852,0 \pm 103,4$ & $7,84 \pm 0,12$ & $3,93 \pm 0,07$ & 11,27 \\
\hline TEPA & $1328,0 \pm 19,8$ & $6,71 \pm 0,03$ & $2,74 \pm 0,05$ & 11,28 \\
\hline AEP & $853,9 \pm 6,1$ & $5,13 \pm 0,01$ & - & 11,14 \\
\hline IPD & $644,9 \pm 0,9$ & $5,06 \pm 0,07$ & - & 11,61 \\
\hline $3 \mathrm{DCM}$ & $439,13 \pm 4,91$ & $5,76 \pm 0,05$ & - & 11,15 \\
\hline
\end{tabular}

Os valores apresentados são resultados da média dos valores encontrados nos procedimentos realizados para cada composto de pelo menos seis análises.
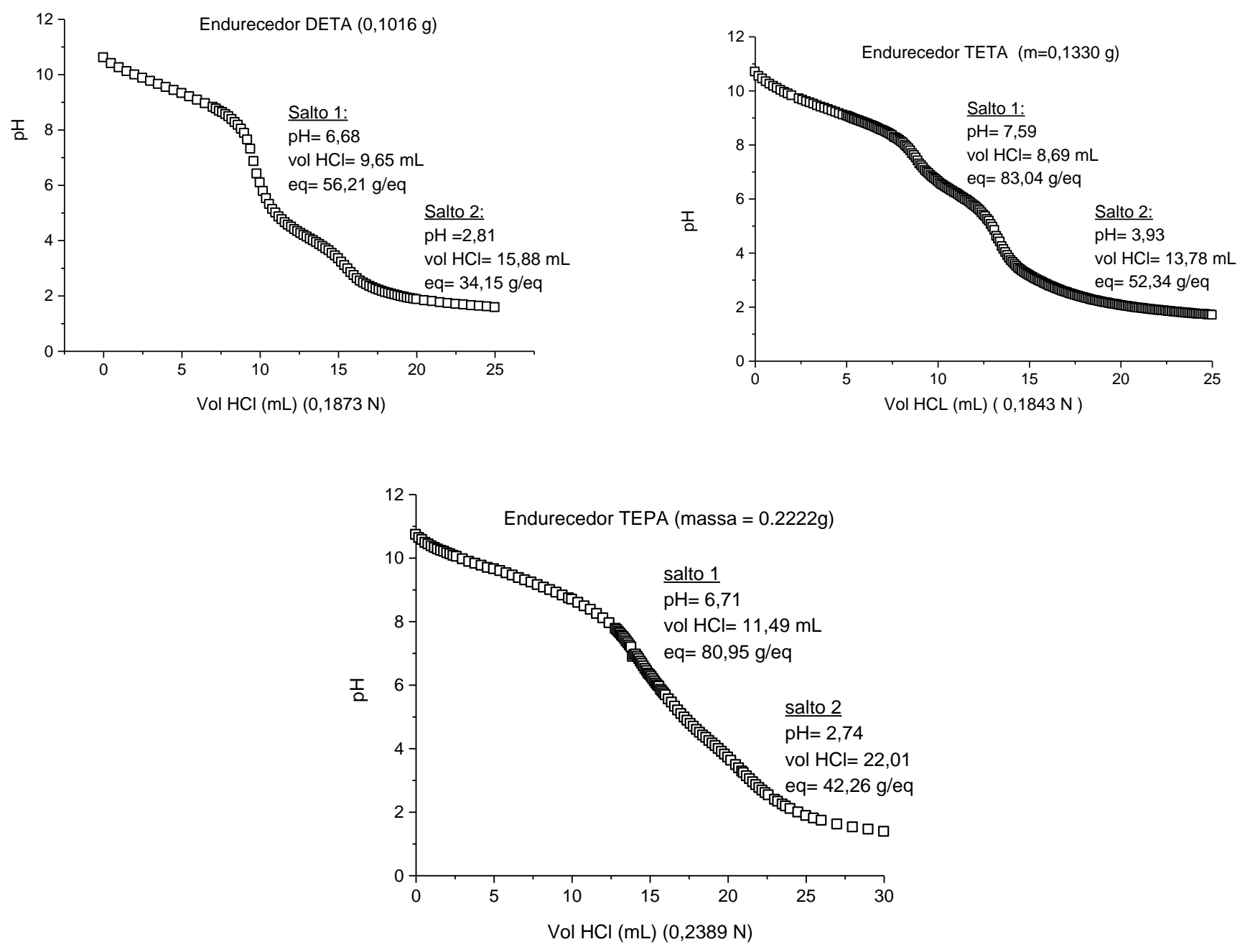

Figura 2: Curvas potenciométricas correspondentes a um processo de titulação em meio aquoso dos endurecedores DETA, TETA, TEPA.

\section{DISCUSSÃO}

A titulação potenciométrica de todos os compostos demonstrou a existência de um ou dois saltos na curva de titulação ( $\mathrm{pH}$ vs Vol. adicionado de solução de $\mathrm{HCl}$, ver Figuras 1 e 2 ). Estes saltos foram relacionados às reações de protonação dos nitrogênios primário, secundário e terciário. $\mathrm{O}$ equivalente de 
ácido consumido no primeiro salto foi atribuído à reação de protonação do nitrogênio primário, devido à maior basicidade quando comparado aos nitrogênios de tipo secundário e terciário, e o segundo salto foi atribuído às reações de protonação dos nitrogênios secundários e terciários de maneira sobreposta. Estes resultados coincidem com os resultados encontrados num trabalho anterior []]. Das titulações é possível obter a concentração de nitrogênios primário e a concentração (soma) dos nitrogênios secundários e terciários (gramas de endurecedor que correspondem a 1 equivalente grama de átomos de nitrogênio).

Para os compostos que apresentaram apenas um salto, EDA, AEP, IPD e 3DCM os valores amínico, de $\mathrm{pH}$ no ponto de equivalência e do $\mathrm{pH}$ da solução aquosa ficaram evidentes. O composto EDA apresentou os maiores valores, exceto quando comparado ao valor do $\mathrm{pH}$ da solução do composto IPD. Isto foi relacionado a um fato bem estabelecido na literatura de que as aminas alifáticas lineares são mais básicas que as aminas cíclicas $[\underline{4}, \underline{8}]$. Para os compostos IPD e 3DCM com estrutura cíclica e que apresentam na sua estrutura dois nitrogênios do tipo primário, foi verificado que o composto IPD resultou mais básico, devido a que mostrou maior valor amínico e maior valor do $\mathrm{pH}$ da solução aquosa como mostra a Tabela 3. No caso, do endurecedor AEP que apresenta uma combinação de estrutura linear e cíclica, este composto parece ser um caso especial, já que apresenta na sua estrutura química os três tipos de nitrogênios, primário, secundário e terciário. Poderíamos esperar dois saltos na curva potenciométrica como nos outros compostos, entretanto foi evidente a existência de apenas um salto. O comportamento mais significativo está relacionado ao maior valor amínico deste composto quando comparado aos compostos IPD e 3DCM. Isto esta justificado pela sua estrutura química, onde o nitrogênio primário esta presente numa estrutura alifática de dois átomos de carbono.

\section{CONCLUSÕES}

Foi obtida de maneira indireta a massa equivalente de hidrogênio ativo do tipo amina dos agentes de cura selecionados. A partir dos dados experimentais coletados nesse trabalho nota-se que mudanças na estrutura química de um composto, resultam em diferentes características físico-químicas. Um outro fato notado no trabalho resulta da pureza dos compostos utilizados, já que as previsões feitas com base nas estruturas químicas básicas dos compostos são bastante sensíveis às composição química.

\section{AGRADECIMENTOS}

Os autores agradecem à FAPEMIG pelo suporte financeiro concedido através de Bolsa de Pósdoutorado (08/07 - 08/08, ref. TEC 00242/07) e; ao CNPq pela Bolsa de iniciação cientifica PIBIC.

\section{BIBLIOGRAFIA}

[1] GARCIA, F.G., MIGUEZ, E., SOARES, B.G., "Caracterização do sistema éter diglicidílico do bisfenol A / poliaminas alifáticas", Polímeros: Ciência e Tecnologia , v. 15, pp. 261-267, 2005.

[2] PASCAUlT, J.P., SAUTEREAU, H., VERDU, J., WILliAMS, R. J.J., Thermosetting Polymers, New York, Marcel Dekker, 2002.

[3] MAY, C.A., Epoxy Resins, Chemistry and Technology, 2 ed., New York, Marcel Dekker. 1988.

[4] LEE, H., NEVILLE, K., Handbook of Epoxy Resins, 1 ed., New York, McGraw-Hill \&Inc. 1967.

[5] Plastics - Amine epoxide hardeners - Determination of primary, secondary and tertiary amine group nitrogen content. International Standard, ISO 9702:1996.

[6] GARCIA, F.G., DA SILVA, P.M., SOARES, B.G., RIEUMONT, J., "Combined analytical techniques for the determination of the amine hydrogen equivalent weight in aliphatic amine epoxide hardeners", Polymer Testing, v. 26, pp. 95-101, 2007.

[7] GARCIA, F.G., TAVAREZ, M.I.B., MIGUEZ E., "Caracterización cualitativa de poliaminas libres en endurecedores de resinas epoxídicas del tipo etilenaminas por espectroscopia de resonancia magnética nuclear", Polímeros: Ciência e Tecnologia, v. 18, pp. 45-51, 2008.

[8] GARCIA, F.G., SAMPAIO, E.M., NEVES, A.F., DE OLIVEIRA, M.G., "Evaluación de la adherencia de uniones adhesivas metálicas con adhesivos epoxídicos modificados", Polímeros: Ciência $e$ Tecnologia, v. 18, pp. 30-44, 2008. 